\title{
chaOPEN
}

\section{Adverse event rates associated with oral anticoagulant treatment early versus later after hospital discharge in older adults: a retrospective population-based cohort study}

\author{
Anne Holbrook MD PharmD, Harsukh Benipal MSc, J. Michael Paterson MSc, Diana Martins MSc, \\ Simon Greaves MSc, Munil Lee BHSc, Tara Gomes PhD MHSc
}

Abstract

Background: Oral anticoagulants are commonly used high-risk medications, but little is known about their safety in transition from hospital to home. Our objective was to measure the rates of hemorrhage and thromboembolic events among older adults receiving oral anticoagulant treatment early after hospital discharge compared to later.

Methods: We conducted a retrospective population-based cohort study among Ontario residents aged 66 years or more who started, continued or resumed oral anticoagulant therapy at hospital discharge between September 2010 and March 2015. We calculated the rates of hemorrhage and thromboembolic events requiring hospital admission or an emergency department visit over a 1-year followup period, stratified by the first 30 days after discharge and the remainder of the year. We used multivariable regression models, adjusting for covariates, to estimate the effect of sex, prevalent versus incident use, and switching anticoagulants on events.

Results: A total of 123139 patients (68 408 women [55.6\%]; mean age 78.2 yr) were included. About one-quarter (32 563 [26.4\%]) had a Charlson Comorbidity Index score of 2 or higher. The rates of hemorrhage and thromboembolic events per 100 person-years were highest during the first 30 days after hospital discharge $(25.8,95 \% \mathrm{Cl} 24.8-26.8$ and $19.3,95 \% \mathrm{Cl} 18.4-20.2$, respectively), falling to $15.7(95 \% \mathrm{Cl} 15.3-16.1)$ and 6.9 (95\% Cl 6.6-7.1), respectively, during the subsequent 11 months. Multivariable analysis showed that patients whose anticoagulant was switched in hospital and men had more hemorrhages and thromboembolic events in follow-up.

Interpretation: The first few weeks following hospital discharge represent a very high-risk period for adverse events related to oral anticoagulant treatment among older adults. The results support an intervention trial addressing anticoagulation management in the early postdischarge period.

ral anticoagulants, including warfarin and the direct-acting anticoagulants, are highly effective for the prevention of stroke and systemic embolism in patients with atrial fibrillation, as well as for the treatment and prevention of venous thromboembolism. ${ }^{1-5}$ Both conditions increase in prevalence with increasing age. ${ }^{6,7}$ More than 7 million prescriptions in Canada and more than 37 million prescriptions in the United States are filled annually for oral anticoagulant treatment. ${ }^{8,9}$ As thromboembolic events increase with increasing age, the absolute risk reduction in events obtained with oral anticoagulant treatment is greater for older adults than for younger people. ${ }^{10-12}$ Despite their benefit, oral anticoagulants are considered high-risk medications because of the risk of substantial harm - mainly bleeding or thromboembolic events, and death - if treatment is not well managed. ${ }^{13}$ Oral anticoagulant treatment has been reported to be the most common drug-related cause of emergency department visits and hospital admission among older adults, with accompanying high mortality rates. ${ }^{14-16}$

The period immediately after hospital discharge can entail high risk for adverse events, as the transition to home is a complex process involving multiple providers, locations, testing and medication changes with imperfect reconciliation at a time when patients are still recovering. In a 2013 study, roughly one-fifth of Medicare patients discharged from hospital

Competing interests: Anne Holbrook has served as an expert policy advisor for national, provincial and local hospital public drug plans for several decades. No other competing interests were declared.

This article has been peer reviewed.

Correspondence to: Anne Holbrook, holbrook@mcmaster.ca CMAJ Open 2021. DOI:10.9778/cmajo.20200138 
required readmission within 30 days. ${ }^{17}$ Studies suggest high rates of medication-related adverse events in the early postdischarge period..$^{18,19}$ Our previous study showed a fourfold greater bleeding risk in older Ontario adults receiving warfarin in the first 30 days after hospital discharge compared to the remainder of the 5 -year follow-up. ${ }^{20}$ Very little is known about the high-risk periods for bleeding or thromboembolic events in the era of direct-acting oral anticoagulant use.

We aimed to measure rates of thromboembolic and bleeding events associated with oral anticoagulant treatment early after hospital discharge (within $30 \mathrm{~d}$ ) compared to the subsequent 11 months. We hypothesized that the early postdischarge period would be associated with a higher risk of adverse events than the later period. Assuming a medicationfocused approach to outcomes as opposed to a disease-specific approach provides a broader view of medication safety.

\section{Methods}

\section{Study design and setting}

We conducted a retrospective population-based cohort study in Ontario, Canada. All Ontario residents have access to publicly funded physician and hospital care, and those aged 65 years or more also have access to prescription medications with a low or no copay. Study methods and reporting followed Strengthening the Reporting of Observational studies in Epidemiology (STROBE) and Reporting of Studies Conducted Using Observational Routinely Collected Health Data
Statement for Pharmacoepidemiology (RECORD-PE) recommendations. ${ }^{21,22}$ A detailed protocol with a prespecified analysis plan was prepared and registered at ICES before data were accessed (Appendix 1, available at www.cmajopen.ca/ content/9/2/E364/suppl/DC1).

\section{Data sources}

Multiple health administrative data sets were linked for this study by means of unique encoded identifiers. Details of the databases and their contents are provided in Table $1 .{ }^{23} \mathrm{In}$ brief, the Ontario Health Insurance Plan database contains billing and diagnostic codes for physician services, the Ontario Drug Benefit program database contains details of outpatient prescription drugs dispensed to those aged 65 or more, and the Canadian Institute for Health Information Discharge Abstract Database and National Ambulatory Care Reporting System detail diagnoses and procedures provided during hospital admissions, and emergency department visits, respectively.

We obtained demographic characteristics and vital status from the Ontario Health Insurance Plan Registered Persons Database. We obtained data on cancer, diabetes, congestive heart failure and hypertension from disease-based registries. ${ }^{24-27} \mathrm{We}$ identified physician specialties from the ICES Physician Database. There is a large literature on the validity and completeness of these population-based databases for identifying drug-related adverse events requiring hospital admission or emergency department visits. ${ }^{28}$

\begin{tabular}{|c|c|}
\hline Name of database & Content \\
\hline $\begin{array}{l}\text { Canadian Institute for Health } \\
\text { Information Discharge Abstract } \\
\text { Database }\end{array}$ & $\begin{array}{l}\text { Patient-level demographic, diagnostic, procedural and treatment information on all } \\
\text { acute care hospital admissions }\end{array}$ \\
\hline $\begin{array}{l}\text { Canadian Institute for Health } \\
\text { Information National Ambulatory } \\
\text { Care Reporting System }\end{array}$ & $\begin{array}{l}\text { Patient-level demographic, diagnostic, procedural and treatment information on all } \\
\text { emergency department visits }\end{array}$ \\
\hline The DrugList File & $\begin{array}{l}\text { List of Drug Identification Numbers used in Canada from } 1990 \text { forward; contains } \\
\text { drug and product names, manufacturer, subclass information, pharmacy } \\
\text { classification group codes, drug strength, route of administration, and first and last } \\
\text { dispensing dates }\end{array}$ \\
\hline ICES-derived cohorts & $\begin{array}{l}\text { Validated cohorts of people with specific diseases and conditions, including the } \\
\text { Ontario Congestive Heart Failure Database, Ontario Diabetes Database and } \\
\text { Ontario Hypertension Data Set }\end{array}$ \\
\hline ICES Physician Database & Characteristics of physicians and surgeons licensed to practise in Ontario \\
\hline Ontario Cancer Registry & $\begin{array}{l}\text { Patient-level demographic information and data on cancer diagnosis and } \\
\text { cancer-related mortality }\end{array}$ \\
\hline $\begin{array}{l}\text { Ontario Drug Benefit program } \\
\text { database }\end{array}$ & $\begin{array}{l}\text { Records of dispensed outpatient prescriptions paid for by the provincial } \\
\text { government }\end{array}$ \\
\hline $\begin{array}{l}\text { Ontario Health Insurance Plan } \\
\text { claims history database }\end{array}$ & Claims for physician services paid for by the provincial government \\
\hline $\begin{array}{l}\text { Ontario Health Insurance Plan } \\
\text { Registered Persons Database }\end{array}$ & $\begin{array}{l}\text { Demographic information and data on place of residence and vital status for all } \\
\text { people eligible to receive insured health care services in the province }\end{array}$ \\
\hline $\begin{array}{l}\text { Statistics Canada Census Postal } \\
\text { Code }\end{array}$ & Information on rural residence and income quintiles of residents \\
\hline
\end{tabular}


All diagnoses were coded with the International Classification of Diseases, 9th Revision or the International Statistical Classification of Diseases and Related Health Problems, 10th Revision. We coded procedures using the Canadian Classification of Interventions and the Canadian Classification of Diagnostic, Therapeutic, and Surgical Procedures. Medications were identified through Health Canada Drug Identification Numbers. A list of codes used in the study is available in Appendix 1.

\section{Participants}

Eligible patients were those who were 66 years of age or more who started, continued or resumed oral anticoagulant therapy after hospital discharge between September 2010 and March 2015. Anticoagulants included warfarin, dabigatran, rivaroxaban and apixaban. We excluded patients who were in their first year of eligibility for provincial prescription drug coverage (age $65 \mathrm{yr}$ ) to avoid incomplete medication records. Patients who had been admitted for a major bleed were excluded, as oral anticoagulant therapy would be contraindicated in many cases. Patients were also excluded if they received more than 1 type of oral anticoagulant at cohort entry or did not have provincial health coverage.

\section{Anticoagulant exposure}

There were 4 oral anticoagulants on the Ontario Drug Benefit program formulary at the time of the study: warfarin and 3 direct-acting drugs (dabigatran, rivaroxaban and apixaban). We defined cohort entry as the dispensing date of the first prescription for oral anticoagulant treatment after hospital discharge in the Ontario Drug Benefit program database. This was captured on the day before, the day of or the day after the hospital discharge date. We defined ongoing use of anticoagulant therapy as successive refills of any prescription for an oral anticoagulant within 30 days or 1.5 times the days' supply of the most recent prescription, whichever was greater. This interval allowed for periodic adjustments to dosages, short pauses and variable timing of refills. If this time frame for refills was exceeded, patients were deemed to have discontinued treatment and were followed for 30 days or 1.5 times the days' supply of their final prescription, whichever was longer.

We classified eligible patients into incident and prevalent users. Incident users were patients who had not been dispensed an oral anticoagulant in the year before cohort entry, and prevalent users were patients who had been dispensed such a medication in that time. Prevalent users were also classified into 2 groups: switchers (those who were receiving a different oral anticoagulant on discharge than just before hospital admission) and nonswitchers (those who continued with the same anticoagulant after discharge as they had been taking before hospital admission).

\section{Outcomes}

Our primary outcomes were hemorrhagic and thromboembolic events requiring admission to hospital or an emergency department visit. We categorized hemorrhages as intracranial, upper or lower gastrointestinal, or other major bleeds based on clinical importance and frequency. ${ }^{29}$ Thromboembolic events included venous events (deep vein thrombosis and pulmonary embolism) and arterial events (ischemic stroke or transient ischemic attack, peripheral vascular disease embolism and systemic embolism). Multiple studies have established the validity of administrative data for identifying hemorrhage and thromboembolic events..$^{30-36}$

Patients were followed until 1 of the following events occurred: death, discontinuation of oral anticoagulant therapy, hospital stay of more than 5 days for reasons other than hemorrhage or a thromboembolic event, 365 days of followup or the end of the study period (Mar. 31, 2016). If a patient had multiple admissions for any outcome of interest during follow-up, we included each event in calculating the rate of events. We assessed major hemorrhagic and thromboembolic events during the postdischarge period at intervals of 0-30 days, 31-364 days and 0-364 days. Figure 1 shows the cohort timeline and definitions.

\section{Variables}

Baseline demographic characteristics included age, sex and rural residence (based on postal code). Data regarding the patients' care included the oral anticoagulant dispensed at the index prescription date and specialty of the physician who wrote the index prescription. Indications for anticoagulant treatment included atrial fibrillation (emergency department visit or hospital admission for atrial fibrillation within the previous $10 \mathrm{yr}$ ); prevention of venous thromboembolism (hip or knee joint replacement within $35 \mathrm{~d}$ before cohort entry, or major surgery during the index hospital stay); treatment of venous thromboembolism (diagnosis of acute deep vein thrombosis or pulmonary embolism during the index hospital stay); or active cancer (codes for cancer-related surgery, chemotherapy or radiation in the Ontario Cancer Registry, Discharge Abstract Database or Ontario Health Insurance Plan database within $180 \mathrm{~d}$ before cohort entry). Patients could have more than 1 indication.

Other past medical history collected at baseline included hemorrhagic and thromboembolic events within the previous 3 years, hospital admissions in the previous year, recent medications that could interact adversely with oral anticoagulant treatment and comorbidity burden (Charlson Comorbidity $\operatorname{Index}^{37}$ ). We calculated individual risks of stroke using the $\mathrm{CHA}_{2} \mathrm{DS}_{2}-\mathrm{VASc}$ score, and risks of major bleeding using the HAS-B_ED score (HAS-BLED without international normalized ratio data), employing previously validated database registries. ${ }^{24,38-41}$

\section{Statistical analysis}

We compared baseline characteristics between incident and prevalent users of oral anticoagulants and, within the prevalent users, between switchers and nonswitchers. We calculated crude rates of hemorrhagic and thromboembolic events during the first 30 days after oral anticoagulant therapy was started, 31-364 days after and the entire year. We used modified intention-to-treat principles for the analysis, meaning that events were attributed to the anticoagulant on the index 


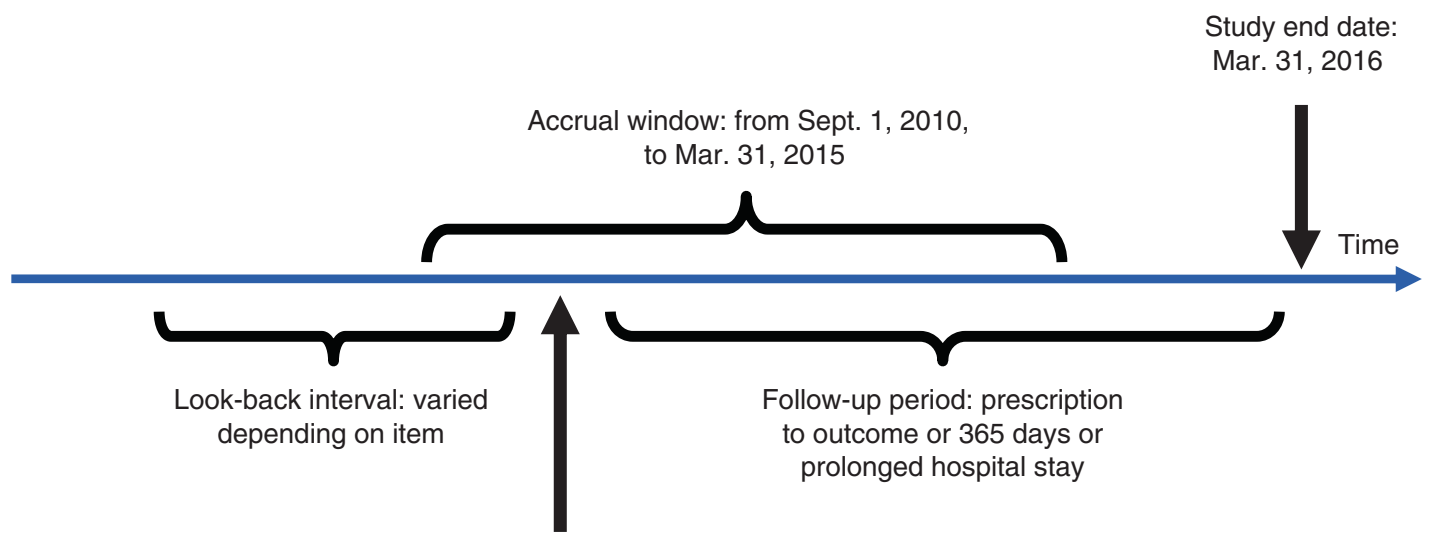

Cohort entry date: ODB prescription dispensed within 1 day of hospital discharge for DOAC or warfarin

Figure 1: Cohort timelines and definitions. Note: ODB = Ontario Drug Benefit program, DOAC = direct-acting oral anticoagulant.

prescription even if the patient switched to another anticoagulant during follow-up. We calculated event rates as the total number of events leading to the hospital admission or emergency department visit for a hemorrhagic or thromboembolic event divided by the person-years available during the interval, stratified by the type of user (incident, switcher or nonswitcher). Multivariable regression models adjusted for sex, age, prevalent versus incident use, warfarin versus directacting anticoagulant use, switch versus no switch, indication for use, Charlson Comorbidity Index score, HAS-B_ED score and $\mathrm{CHA}_{2} \mathrm{DS}_{2}-\mathrm{VASc}$ score.

\section{Ethics approval}

The use of data in this project was authorized under section 45 of Ontario's Personal Health Information Protection Act, which does not require review by a research ethics board.

\section{Results}

We assessed 3036285 patients who were discharged from an Ontario hospital during the accrual period for eligibility. Once exclusions were made for missing identifiers, ineligible age, no prescription for oral anticoagulant treatment within 1 day after hospital admission, death before cohort entry and duplicate prescription for multiple oral anticoagulants, 123139 eligible patients were identified (Figure 2).

Table 2 shows the baseline characteristics of the cohort and subgroups. The patients' mean age was 78.2 (standard deviation [SD] 7.7) years. Of the 123139 patients, 68408
(55.6\%) were women, and 19931 (16.2\%) resided in a rural area. Indications for oral anticoagulant treatment included atrial fibrillation (62 957 patients [51.1\%]), recent joint replacement (44 375 [36.0\%]), major surgery during the index hospital stay (22 043 [17.9\%]), active cancer (7858 [6.4\%]), and deep vein thrombosis or pulmonary embolism diagnosed during the index hospital stay (6407 [5.2\%]). Patients were most commonly dispensed warfarin (59232 [48.1\%]) or rivaroxaban (51 409 [41.7\%]. A total of 70140 patients $(57.0 \%)$ were incident users, and 52999 (43.0\%) were prevalent users. Prevalent users were older than incident users (mean age 81.1 [SD 7.6] yr v. 76.1 [SD 7.1] yr), and a higher proportion received their index prescription from their family physician (28 920 [54.6\%] v. 12604 [18.0\%]).

Of the 52999 prevalent users, 49325 (93.1\%) were nonswitchers, and 3674 (6.9\%) were switchers. A total of 1481 of the switchers (40.3\%) switched from a direct-acting anticoagulant to warfarin, and 2193 (59.7\%) switched from warfarin to a direct-acting anticoagulant.

There were 9784 deaths $(7.9 \%)$ over the year of followup. Rates of major hemorrhage per 100 person-years declined from 25.8 (95\% CI 24.8-26.8) in the first 30 days after hospital discharge to 15.7 (95\% CI 15.3-16.1) over the subsequent 11 months (Table 3, Figure 3). Upper gastrointestinal bleeds were the most common type of specified bleed, with an annual rate of 4.8 per 100 person-years $(95 \%$ CI 4.6-5.0). Prevalent users experienced a higher overall rate of hemorrhage per 100 person-years than incident users (20.4, 95\% CI 19.9-20.9 v. 14.6, 95\% CI 14.1-15.1) (Table 4). 


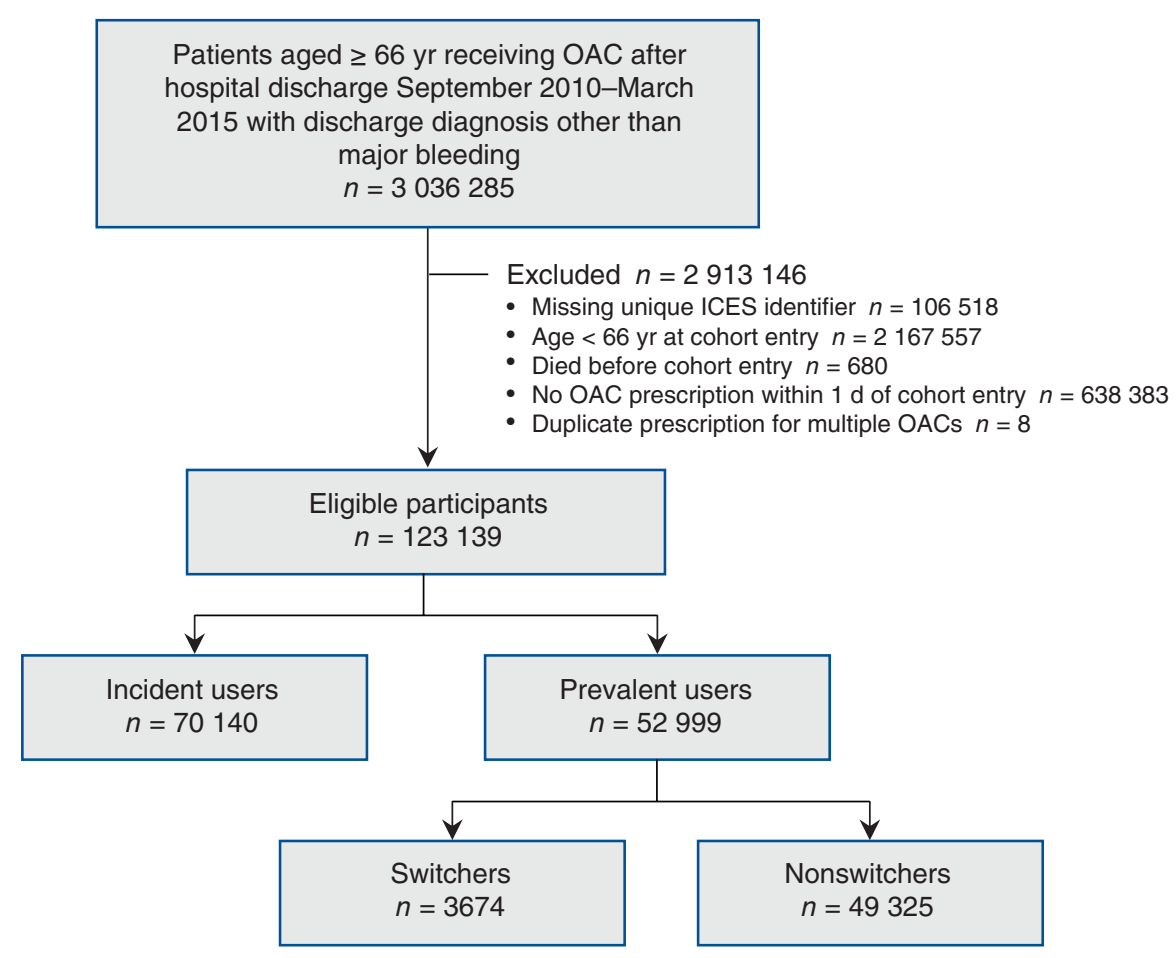

Figure 2: Flow diagram showing participant selection. Note: $\mathrm{OAC}=$ oral anticoagulant.

However, after adjustment for covariates, this difference decreased to an estimated incidence rate ratio (IRR) of 1.01 (95\% CI 0.95-1.08). Among prevalent users, the rate of hemorrhage was higher for switchers than for nonswitchers (IRR 1.17, 95\% CI 1.02-1.33) (Table 5). Men were more likely than women to experience hemorrhage (21.4 per 100 person-years, $95 \%$ CI 20.8-22.0 v. 14.8 per 100 personyears, 95\% CI 14.4-15.3), with an IRR of 1.32 (95\% CI 1.26-1.40) (Table 6). Choice of anticoagulant (warfarin v. direct-acting) at discharge did not predict the hemorrhage rate (IRR 1.00, 95\% CI 0.94-1.06).

The rate of thromboembolic events per 100 person-years decreased from 19.3 (95\% CI 18.4-20.2) in the first 30 days after discharge to 6.9 (95\% CI 6.6-7.1) over the subsequent 11 months (Table 3, Figure 4). Of the 4643 events over the year, 2485 (53.5\%) were arterial, including 1696 ischemic strokes or transient ischemic attacks or systemic embolisms (36.5\%), compared to 1180 deep vein thromboses $(25.4 \%)$ and 978 pulmonary embolisms $(21.1 \%)$, representing venous events. The overall rate of thromboembolic events per 100 person-years was higher among incident users than among prevalent users (10.0, 95\% CI 9.6-10.4 v. 8.9, 95\% CI 8.5-9.2) (Table 4). Although incident users were more likely to have had joint replacement surgery during the index hospi- tal stay and to have lower Charlson Comorbidity Index scores (therefore a higher odds of no events [OR 3.94, 95\% CI 3.304.69]), those who did have an event were likely to have more events (IRR 3.04, 95\% CI 2.75-3.36). After adjustment for the other covariates, switchers were more likely than nonswitchers to have thromboembolic events (IRR 1.30, 95\% CI 1.08-1.55) (Table 5). Men had a higher rate of thromboembolic events than women (IRR 1.13, 95\% CI 1.04-1.23) (Table 6). Warfarin was associated with more thromboembolic events than the direct-acting anticoagulants (IRR 1.16, 95\% CI 1.07-1.26).

\section{Interpretation}

In this population-based cohort study involving older adults in Ontario, rates of hemorrhage and thromboembolic events were very high in the first 30 days after hospital discharge, considerably higher than in the subsequent 11 months. Although incident users included a large number of shortterm users (e.g., venous thromboembolism prophylaxis after orthopedic surgery), their 30-day event rates per 100 personyears were still high, at 21.4 (95\% CI 20.2-22.6) for thromboembolic events and 21.9 (95\% CI 20.7-23.1) for hemorrhagic events. Prevalent users were more likely than incident users to 
Table 2 (part 1 of 2): Baseline characteristics of older adults in Ontario who started, continued or resumed oral anticoagulant therapy after hospital discharge between September 2010 and March 2015

\begin{tabular}{|c|c|c|c|c|c|c|c|}
\hline \multirow[b]{2}{*}{ Characteristic } & \multicolumn{4}{|c|}{ Entire cohort; no (\%) of patients* } & \multicolumn{3}{|c|}{ Prevalent users; no (\%) of patients ${ }^{*}$} \\
\hline & $\begin{array}{c}\text { Overall } \\
n=123139\end{array}$ & $\begin{array}{c}\text { Incident users } \\
n=70140\end{array}$ & $\begin{array}{l}\text { Prevalent users } \\
n=52999\end{array}$ & $\begin{array}{l}\text { Standardized } \\
\text { difference }\end{array}$ & $\begin{array}{l}\text { Switchers } \\
n=3674\end{array}$ & $\begin{array}{c}\text { Nonswitchers } \\
n=49325\end{array}$ & $\begin{array}{l}\text { Standardized } \\
\text { difference }\end{array}$ \\
\hline Age, mean $\pm S D, y r$ & $78.2 \pm 7.7$ & $76.1 \pm 7.1$ & $81.1 \pm 7.6$ & 0.69 & $79.4 \pm 7.3$ & $81.23 \pm 7.6$ & 0.24 \\
\hline Female sex & $68408(55.6)$ & $39956(57.0)$ & $28452(53.7)$ & 0.07 & $1846(50.2)$ & $26606(53.9)$ & 0.07 \\
\hline Rural residence $\dagger$ & $19931(16.2)$ & $11892(17.0)$ & $8039(15.2)$ & 0.05 & $580(15.8)$ & $7459(15.1)$ & 0.02 \\
\hline \multicolumn{8}{|l|}{ Anticoagulant dispensed } \\
\hline Apixaban & $5890(4.8)$ & $2810(4.0)$ & $3080(5.8)$ & 0.08 & $570(15.5)$ & $2510(5.1)$ & 0.35 \\
\hline Dabigatran & $6608(5.4)$ & $2775(4.0)$ & $3833(7.2)$ & 0.14 & $473(12.9)$ & $3360(6.8)$ & 0.20 \\
\hline Rivaroxaban & $51409(41.7)$ & $42546(60.7)$ & $8863(16.7)$ & 1.01 & $1150(31.3)$ & $7713(15.6)$ & 0.38 \\
\hline Warfarin & $59232(48.1)$ & 22009 (31.4) & $37223(70.2)$ & 0.84 & $1481(40.3)$ & $35742(72.5)$ & 0.69 \\
\hline \multicolumn{8}{|l|}{ Indication $\ddagger$} \\
\hline Atrial fibrillation within $10 \mathrm{yr}$ & $62957(51.1)$ & $22530(32.1)$ & $40427(76.3)$ & 0.99 & $2988(81.3)$ & $37439(75.9)$ & 0.13 \\
\hline Joint replacement within $35 \mathrm{~d}$ & $44375(36.0)$ & 38939 (55.5) & $5436(10.3)$ & 1.10 & $502(13.7)$ & $4934(10.0)$ & 0.11 \\
\hline $\begin{array}{l}\text { Major surgery during index } \\
\text { hospital stay }\end{array}$ & $22043(17.9)$ & $17384(24.8)$ & $4659(8.8)$ & 0.44 & $590(16.1)$ & $4069(8.2)$ & 0.24 \\
\hline Active cancer within $180 \mathrm{~d}$ & $7858(6.4)$ & $3548(5.1)$ & $4310(8.1)$ & 0.12 & $278(7.6)$ & $4032(8.2)$ & 0.02 \\
\hline $\begin{array}{l}\text { Deep vein thrombosis or } \\
\text { pulmonary embolism during } \\
\text { index hospital stay }\end{array}$ & $6407(5.2)$ & $1783(2.5)$ & $4624(8.7)$ & 0.27 & $349(9.5)$ & $4275(8.7)$ & 0.03 \\
\hline \multicolumn{8}{|l|}{$\begin{array}{l}\text { Prescribing physician } \\
\text { specialty } \dagger\end{array}$} \\
\hline Family medicine & $41524(33.7)$ & $12604(18.0)$ & $28920(54.6)$ & 0.82 & $1274(34.7)$ & $27646(56.0)$ & 0.44 \\
\hline Orthopedic surgery & $31394(25.5)$ & 28014 (39.9) & $3380(6.4)$ & 0.87 & $287(7.8)$ & $3093(6.3)$ & 0.06 \\
\hline Internal medicine & $9958(8.1)$ & $5350(7.6)$ & $4608(8.7)$ & 0.04 & $432(11.8)$ & $4176(8.5)$ & 0.11 \\
\hline Cardiology & $7083(5.8)$ & $3840(5.5)$ & $3243(6.1)$ & 0.03 & $441(12.0)$ & $2802(5.7)$ & 0.22 \\
\hline Hematology & $2324(1.9)$ & $1808(2.6)$ & $516(1.0)$ & 0.12 & $107(2.9)$ & $409(0.8)$ & 0.15 \\
\hline Other & $8792(7.1)$ & $4843(6.9)$ & $3949(7.5)$ & 0.02 & $387(10.5)$ & $3562(7.2)$ & 0.12 \\
\hline Unknown & 22064 (17.9) & $13681(19.5)$ & $8383(15.8)$ & 0.10 & $746(20.3)$ & $7637(15.5)$ & 0.13 \\
\hline \multicolumn{8}{|l|}{ Past medical history } \\
\hline $\begin{array}{l}\text { No. of hospital admissions } \\
\text { within } 1 \mathrm{yr} \text {, mean } \pm \mathrm{SD}\end{array}$ & $0.67 \pm 1.16$ & $0.30 \pm 0.73$ & $1.16 \pm 1.42$ & 0.76 & $0.99 \pm 1.32$ & $1.17 \pm 1.43$ & 0.13 \\
\hline $\begin{array}{l}\text { No. of thromboembolic events } \\
\text { within } 3 \mathrm{yr}\end{array}$ & 13741 (11.2) & $10483(19.8)$ & $3258(4.6)$ & 0.48 & $730(19.9)$ & $9753(19.8)$ & 0.00 \\
\hline Ischemic stroke & $4419(3.6)$ & $990(1.4)$ & $3429(6.5)$ & 0.26 & $228(6.2)$ & $3201(6.5)$ & 0.01 \\
\hline Transient ischemic attack & $2757(2.2)$ & $853(1.2)$ & $1904(3.6)$ & 0.16 & $142(3.9)$ & $1762(3.6)$ & 0.02 \\
\hline $\begin{array}{l}\text { Peripheral vascular disease } \\
\text { event }\end{array}$ & $2540(2.1)$ & $680(1.0)$ & $1860(3.5)$ & 0.17 & $106(2.9)$ & $1754(3.6)$ & 0.04 \\
\hline Systemic embolism & $705(0.6)$ & $155(0.2)$ & $550(1.0)$ & 0.10 & $34(0.9)$ & $516(1.0)$ & 0.01 \\
\hline Pulmonary embolism & $2393(1.9)$ & $349(0.5)$ & $2044(3.9)$ & 0.23 & $152(4.1)$ & $1892(3.8)$ & 0.02 \\
\hline Deep vein thrombosis & $3280(2.7)$ & $580(0.8)$ & $2700(5.1)$ & 0.25 & $204(5.6)$ & $2496(5.1)$ & 0.02 \\
\hline Hemorrhagic event within $3 \mathrm{yr}$ & $13406(10.9)$ & $3627(5.2)$ & $9779(18.5)$ & 0.42 & $616(16.8)$ & $9163(18.6)$ & 0.05 \\
\hline Intracranial bleeding & $777(0.6)$ & $230(0.3)$ & $547(1.0)$ & 0.09 & $27(0.7)$ & $520(1.1)$ & 0.03 \\
\hline Upper gastrointestinal bleeding & $3830(3.1)$ & $1068(1.5)$ & $2762(5.2)$ & 0.21 & $182(5.0)$ & $2580(5.2)$ & 0.01 \\
\hline Lower gastrointestinal bleeding & $1498(1.2)$ & $453(0.6)$ & $1045(2.0)$ & 0.12 & $85(2.3)$ & $960(1.9)$ & 0.03 \\
\hline Other major bleed & $8750(7.1)$ & $2132(3.0)$ & $6618(12.5)$ & 0.36 & $392(10.7)$ & $6226(12.6)$ & 0.06 \\
\hline
\end{tabular}


Table 2 (part 2 of 2): Baseline characteristics of older adults in Ontario who started, continued or resumed oral anticoagulant therapy after hospital discharge between September 2010 and March 2015

\begin{tabular}{|c|c|c|c|c|c|c|c|}
\hline \multirow[b]{2}{*}{ Characteristic } & \multicolumn{4}{|c|}{ Entire cohort; no (\%) of patients* } & \multicolumn{3}{|c|}{ Prevalent users; no (\%) of patients* } \\
\hline & $\begin{array}{c}\text { Overall } \\
n=123139\end{array}$ & $\begin{array}{l}\text { Incident users } \\
n=70140\end{array}$ & $\begin{array}{l}\text { Prevalent users } \\
n=52999\end{array}$ & $\begin{array}{l}\text { Standardized } \\
\text { difference }\end{array}$ & $\begin{array}{l}\text { Switchers } \\
n=3674\end{array}$ & $\begin{array}{c}\text { Nonswitchers } \\
n=49325\end{array}$ & $\begin{array}{l}\text { Standardized } \\
\text { difference }\end{array}$ \\
\hline \multicolumn{8}{|l|}{ Comorbidities } \\
\hline Congestive heart failure & $47133(38.3)$ & 14265 (20.3) & $32868(62.0)$ & 0.93 & $2096(57.0)$ & $30772(62.4)$ & 0.11 \\
\hline Hypertension & $106292(86.3)$ & 57447 (81.9) & $48845(92.2)$ & 0.31 & $3378(91.9)$ & 45467 (92.2) & 0.01 \\
\hline Diabetes & $46522(37.8)$ & 22569 (32.2) & $23953(45.2)$ & 0.27 & $1627(44.3)$ & $22326(45.3)$ & 0.02 \\
\hline Renal dysfunction§ & $11216(9.1)$ & $2491(3.6)$ & $8725(16.5)$ & 0.44 & $418(11.4)$ & $8307(16.8)$ & 0.16 \\
\hline Liver dysfunctionף & $1349(1.1)$ & $343(0.5)$ & $1006(1.9)$ & 0.13 & $68(1.9)$ & $938(1.9)$ & 0.00 \\
\hline Drug use disorder & $14226(11.6)$ & $11642(16.6)$ & $2584(4.9)$ & 0.39 & $202(5.5)$ & $2382(4.8)$ & 0.03 \\
\hline $\begin{array}{l}\text { Alcohol use disorder in previous } \\
3 \mathrm{yr}\end{array}$ & $1401(1.1)$ & $517(0.7)$ & $884(1.7)$ & 0.09 & $64(1.7)$ & $820(1.7)$ & 0.01 \\
\hline \multicolumn{8}{|l|}{$\begin{array}{l}\text { Charlson Comorbidity Index } \\
\text { score }\end{array}$} \\
\hline 0 & $20946(17.0)$ & $11714(16.7)$ & $9232(17.4)$ & 0.02 & $669(18.2)$ & $8563(17.4)$ & 0.02 \\
\hline 1 & $14766(12.0)$ & $6041(8.6)$ & $8725(16.5)$ & 0.24 & $637(17.3)$ & $8088(16.4)$ & 0.03 \\
\hline$\geq 2$ & $32563(26.4)$ & 8967 (12.8) & $23596(44.5)$ & 0.75 & $1355(36.9)$ & $22241(45.1)$ & 0.17 \\
\hline NA (no hospital admission) & $54864(44.6)$ & $43418(61.9)$ & $11446(21.6)$ & 0.90 & $1013(27.6)$ & $10433(21.2)$ & 0.15 \\
\hline \multicolumn{8}{|l|}{$\mathrm{CHA}_{2} \mathrm{DS}_{2}$-VASc score } \\
\hline Mean \pm SD & $4.08 \pm 1.59$ & $3.49 \pm 1.37$ & $4.86 \pm 1.53$ & 0.95 & $4.77 \pm 1.47$ & $4.87 \pm 1.53$ & 0.07 \\
\hline Median (IQR) & $4(3-5)$ & $3(3-4)$ & $5(4-6)$ & 0.98 & $5(4-6)$ & $5(4-6)$ & 0.07 \\
\hline \multicolumn{8}{|l|}{ HAS-B_ED score } \\
\hline Mean \pm SD & $2.20 \pm 0.68$ & $2.09 \pm 0.63$ & $2.36 \pm 0.71$ & 0.41 & $2.30 \pm 0.69$ & $2.37 \pm 0.72$ & 0.09 \\
\hline Median (IQR) & $2(2-3)$ & $2(2-2)$ & $2(2-3)$ & 0.38 & $2(2-3)$ & $2(2-3)$ & 0.10 \\
\hline \multicolumn{8}{|l|}{$\begin{array}{l}\text { Concomitant medication } \\
\text { within previous } 120 \mathrm{~d}\end{array}$} \\
\hline $\begin{array}{l}\text { Nonsteroidal anti-inflammatory } \\
\text { drug }\end{array}$ & $19273(15.7)$ & 15344 (21.9) & $3929(7.4)$ & 0.42 & $304(8.3)$ & $3625(7.3)$ & 0.03 \\
\hline Acetylsalicylic acid & $2870(2.3)$ & $2212(3.2)$ & $658(1.2)$ & 0.13 & $41(1.1)$ & $617(1.3)$ & 0.01 \\
\hline Other antiplatelet & $7026(5.7)$ & $4459(6.4)$ & $2567(4.8)$ & 0.07 & $207(5.6)$ & $2360(4.8)$ & 0.04 \\
\hline Amiodarone & 4048 (3.3) & $598(0.9)$ & $3450(6.5)$ & 0.30 & $242(6.6)$ & $3208(6.5)$ & 0.00 \\
\hline $\begin{array}{l}\text { Selective serotonin reuptake } \\
\text { inhibitor }\end{array}$ & $14864(12.1)$ & $6189(8.8)$ & 8675 (16.4) & 0.23 & $428(11.6)$ & $8247(16.7)$ & 0.15 \\
\hline Antibiotic ${ }^{\star *}$ & $17345(14.1)$ & $7384(10.5)$ & $9961(18.8)$ & 0.24 & $541(14.7)$ & $9420(19.1)$ & 0.12 \\
\hline \multicolumn{8}{|c|}{$\begin{array}{l}\text { Note: IQR = interquartile range, } \mathrm{NA}=\text { not available, } \mathrm{SD}=\text { standard deviation. } \\
\text { *Except where noted otherwise. } \\
\text { †Data missing for less than } 0.07 \% \text { of patients. } \\
\text { †Patients could have more than } 1 \text { indication. } \\
\text { §Included International Statistical Classification of Diseases and Related Health Problems, 10th Revision (ICD-10) codes for dialysis, chronic renal disease, renal cancer and } \\
\text { renal surgery. } \\
\text { शIncluded ICD-10 codes for cirrhosis, chronic liver disease, liver cancer, hepatitis and liver surgery. } \\
\star * \text { Within } 30 \text { days. }\end{array}$} \\
\hline
\end{tabular}

experience a hemorrhagic event at any time point but were less likely to experience a thromboembolic event. Event rates throughout the follow-up period were significantly higher among men than among women, and among prevalent users who switched anticoagulant agents than among those who did not switch. The mortality rate during the year of follow-up in our cohort, $7.9 \%$, was relatively high.
An overview of event rates from randomized trials in patients with venous thromboembolism showed a rate of recurrent thromboembolism of $1 \%$ and a rate of major bleeding of $0.7 \%$ at 3 months while patients were receiving oral anticoagulant treatment. ${ }^{42}$ In patients with atrial fibrillation, the stroke rate without anticoagulation has been reported to be about $4 \%-5 \%$ per 100 person-years, compared to a 


\begin{tabular}{|c|c|c|c|c|}
\hline \multirow[b]{2}{*}{ Outcome } & \multirow{2}{*}{$\begin{array}{l}\text { No. }(\%) \text { of } \\
\text { patients }\end{array}$} & \multicolumn{3}{|c|}{ Time; event rate per 100 person-years $(95 \% \mathrm{Cl})$} \\
\hline & & $1 \mathrm{yr}$ & First $30 \mathrm{~d}$ & $2-12 \mathrm{mo}$ \\
\hline Hemorrhage & $8767(100.0)$ & $17.7(17.4-18.1)$ & $25.8(24.8-26.8)$ & $15.7(15.3-16.1)$ \\
\hline Intracranial bleed & $664(7.6)$ & $1.3(1.2-1.4)$ & $1.2(1.0-1.4)$ & $1.4(1.3-1.5)$ \\
\hline Upper gastrointestinal bleed & $2392(27.3)$ & $4.8(4.6-5.0)$ & $7.5(7.0-8.0)$ & $4.2(4.0-4.4)$ \\
\hline Lower gastrointestinal bleed & $669(7.6)$ & $1.4(1.3-1.5)$ & $1.9(1.6-2.2)$ & $1.2(1.1-1.3)$ \\
\hline Other major bleed & $5042(57.5)$ & $10.2(9.9-10.5)$ & $15.3(14.5-16.0)$ & $8.9(8.6-9.2)$ \\
\hline Thromboembolic event & $4643(100.0)$ & $9.4(9.1-9.7)$ & $19.3(18.4-20.2)$ & $6.9(6.6-7.1)$ \\
\hline Ischemic stroke & $1001(21.6)$ & $2.0(1.9-2.2)$ & $2.8(2.5-3.2)$ & $1.8(1.7-2.0)$ \\
\hline Transient ischemic attack & $542(11.7)$ & $1.1(1.0-1.2)$ & $1.5(1.2-1.7)$ & $1.0(0.9-1.1)$ \\
\hline Peripheral vascular disease & $789(17.0)$ & $1.6(1.5-1.7)$ & $1.9(1.6-2.1)$ & $1.5(1.4-1.7)$ \\
\hline Systemic embolism & $153(3.3)$ & $0.3(0.3-0.4)$ & $0.6(0.5-0.8)$ & $0.2(0.2-0.3)$ \\
\hline Pulmonary embolism & $978(21.1)$ & $2.0(1.9-2.1)$ & $6.4(5.9-6.9)$ & $0.9(0.8-1.0)$ \\
\hline Deep vein thrombosis & $1180(25.4)$ & $2.4(2.3-2.5)$ & $6.2(5.7-6.7)$ & $1.4(1.3-1.5)$ \\
\hline
\end{tabular}

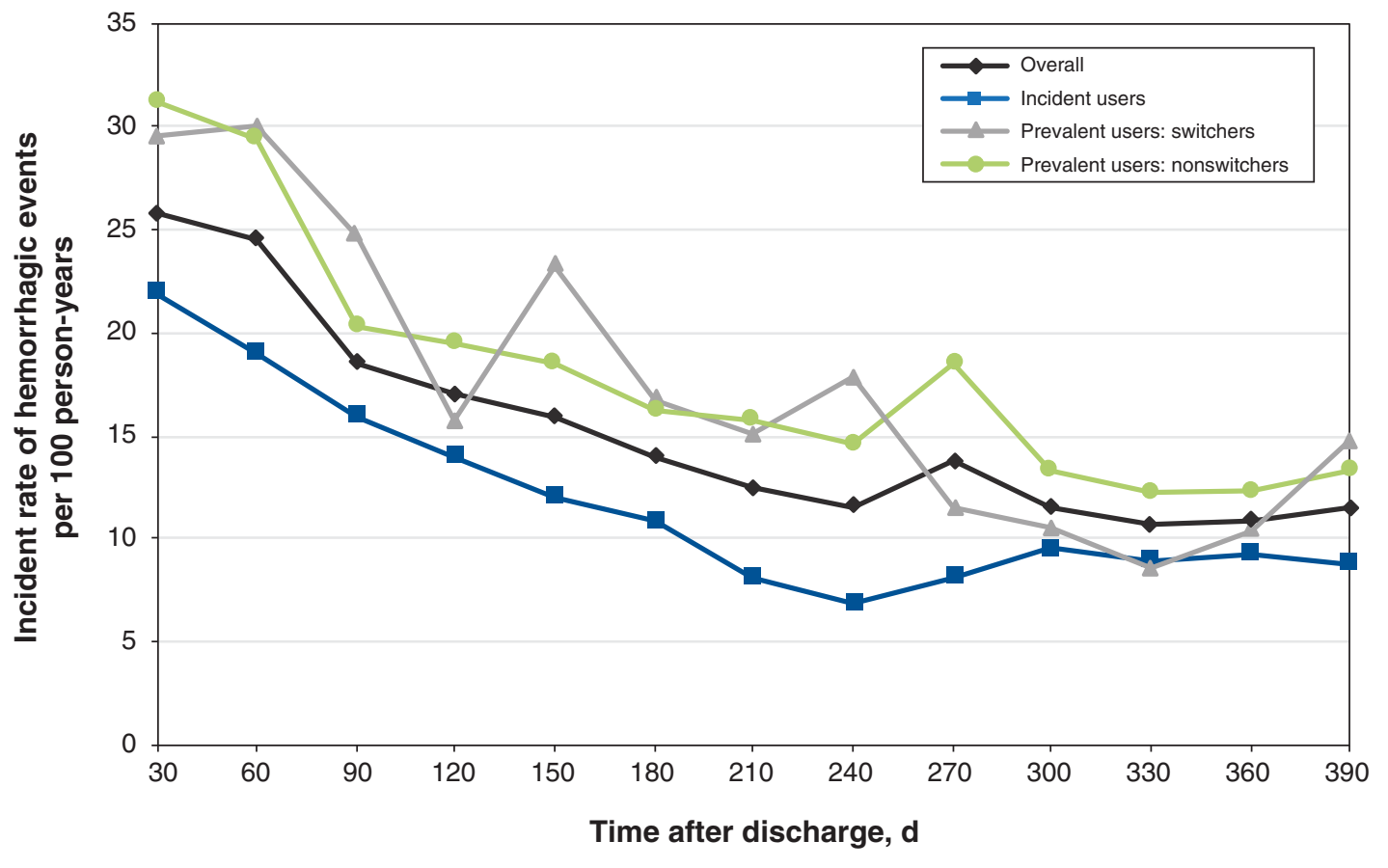

Figure 3: Postdischarge hemorrhage event rates.

bleeding rate of $3.5 \%-3.8 \%$ per 100 person-years. ${ }^{43,44}$ These rates are less than one-third of the early postdischarge rates in our study.

Our results support the contention that transitions in care for patients should be a target for research on interventions intended to lower adverse outcome rates. ${ }^{45,46}$ A 2014 systematic review showed that frequent patient contact, dedicated teams for discharge planning and home visits were most effective at reducing early readmissions. ${ }^{47}$ In the Canadian context, a large cohort study showed that rates of 30-day nonelective 


\begin{tabular}{|c|c|c|c|c|c|c|c|c|}
\hline \multirow[b]{3}{*}{ Outcome } & \multicolumn{4}{|c|}{ Incident users } & \multicolumn{4}{|c|}{ Prevalent users } \\
\hline & \multirow{2}{*}{$\begin{array}{l}\text { No. }(\%) \text { of } \\
\text { patients }\end{array}$} & \multicolumn{3}{|c|}{$\begin{array}{l}\text { Time; event rate per } 100 \text { person-years } \\
\qquad(95 \% \mathrm{Cl})\end{array}$} & \multirow{2}{*}{$\begin{array}{l}\text { No. }(\%) \text { of } \\
\text { patients }\end{array}$} & \multicolumn{3}{|c|}{$\begin{array}{l}\text { Time; event rate per } 100 \text { person-years } \\
\qquad(95 \% \mathrm{Cl})\end{array}$} \\
\hline & & $1 \mathrm{yr}$ & First $30 \mathrm{~d}$ & $2-12 \mathrm{mo}$ & & $1 \mathrm{yr}$ & First $30 \mathrm{~d}$ & 2-12 mo \\
\hline Hemorrhage & $3312(37.8)$ & $\begin{array}{c}14.6 \\
(14.1-15.1)\end{array}$ & $\begin{array}{c}21.9 \\
(20.7-23.1)\end{array}$ & $\begin{array}{c}12.1 \\
(11.6-12.7)\end{array}$ & 5455 (62.2) & $\begin{array}{c}20.4 \\
(19.9-20.9)\end{array}$ & $\begin{array}{c}31.1 \\
(29.4-32.8)\end{array}$ & $\begin{array}{c}18.4 \\
(17.8-18.9)\end{array}$ \\
\hline $\begin{array}{l}\text { Thromboembolic } \\
\text { event }\end{array}$ & $2274(49.0)$ & $\begin{array}{c}10.0 \\
(9.6-10.4)\end{array}$ & $\begin{array}{c}21.4 \\
(20.2-22.6)\end{array}$ & $\begin{array}{c}6.2 \\
(5.8-6.7)\end{array}$ & $2369(51.0)$ & $\begin{array}{c}8.9 \\
(8.5-9.2)\end{array}$ & $\begin{array}{c}16.5 \\
(15.3-17.7)\end{array}$ & $\begin{array}{c}7.4 \\
(7.0-7.8)\end{array}$ \\
\hline
\end{tabular}

\begin{tabular}{|c|c|c|c|c|c|c|c|c|}
\hline \multirow[b]{3}{*}{ Outcome } & \multicolumn{4}{|c|}{ Nonswitchers } & \multicolumn{4}{|c|}{ Switchers } \\
\hline & \multirow{2}{*}{$\begin{array}{l}\text { No. }(\%) \text { of } \\
\text { patients }\end{array}$} & \multicolumn{3}{|c|}{$\begin{array}{l}\text { Time; event rate per } 100 \text { person-years } \\
\qquad(95 \% \mathrm{Cl})\end{array}$} & \multirow{2}{*}{$\begin{array}{l}\text { No. }(\%) \text { of } \\
\text { patients }\end{array}$} & \multicolumn{3}{|c|}{$\begin{array}{l}\text { Time; event rate per } 100 \text { person-years } \\
\qquad(95 \% \mathrm{Cl})\end{array}$} \\
\hline & & $1 \mathrm{yr}$ & First $30 \mathrm{~d}$ & $2-12 \mathrm{mo}$ & & $1 \mathrm{yr}$ & First $30 \mathrm{~d}$ & $2-12$ mo \\
\hline Hemorrhage & $5044(92.5)$ & $\begin{array}{c}20.5 \\
(19.9-21.0)\end{array}$ & $\begin{array}{c}31.2 \\
(29.5-32.9)\end{array}$ & $\begin{array}{c}18.4 \\
(17.8-19.0)\end{array}$ & $411(7.5)$ & $\begin{array}{c}19.6 \\
(17.7-21.5)\end{array}$ & $\begin{array}{c}29.5 \\
(23.4-35.7)\end{array}$ & $\begin{array}{c}18.0 \\
(16.0-19.9)\end{array}$ \\
\hline $\begin{array}{l}\text { Thromboembolic } \\
\text { event }\end{array}$ & $2164(91.3)$ & $\begin{array}{c}8.8 \\
(8.4-9.2)\end{array}$ & $\begin{array}{c}16.0 \\
(14.7-17.2)\end{array}$ & $\begin{array}{c}7.4 \\
(7.0-7.8)\end{array}$ & 205 (8.7) & $\begin{array}{c}9.8 \\
(8.5-11.1)\end{array}$ & $\begin{array}{c}23.8 \\
(18.3-29.4)\end{array}$ & $\begin{array}{c}7.5 \\
(6.2-8.7)\end{array}$ \\
\hline
\end{tabular}

\begin{tabular}{|c|c|c|c|c|c|c|c|c|}
\hline \multirow[b]{3}{*}{ Outcome } & \multicolumn{4}{|c|}{ Men } & \multicolumn{4}{|c|}{ Women } \\
\hline & \multirow{2}{*}{$\begin{array}{l}\text { No. }(\%) \text { of } \\
\text { patients }\end{array}$} & \multicolumn{3}{|c|}{$\begin{array}{l}\text { Time; event rate per } 100 \text { person-years } \\
(95 \% \mathrm{Cl})\end{array}$} & \multirow{2}{*}{$\begin{array}{l}\text { No. }(\%) \text { of } \\
\text { patients }\end{array}$} & \multicolumn{3}{|c|}{$\begin{array}{l}\text { Time; event rate per } 100 \text { person-years } \\
\qquad(95 \% \mathrm{Cl})\end{array}$} \\
\hline & & $1 \mathrm{yr}$ & First $30 \mathrm{~d}$ & $2-12 \mathrm{mo}$ & & $1 \mathrm{yr}$ & First $30 \mathrm{~d}$ & 2-12 mo \\
\hline Hemorrhage & 4677 (53.3) & $\begin{array}{c}21.4 \\
(20.8-22.0)\end{array}$ & $\begin{array}{c}32.1 \\
(30.5-33.8)\end{array}$ & $\begin{array}{c}18.7 \\
(18.0-19.3)\end{array}$ & $4090(46.7)$ & $\begin{array}{c}14.8 \\
(14.4-15.3)\end{array}$ & $\begin{array}{c}20.8 \\
(19.6-22.0)\end{array}$ & $\begin{array}{c}13.3 \\
(12.9-13.8)\end{array}$ \\
\hline $\begin{array}{l}\text { Thromboembolic } \\
\text { event }\end{array}$ & 2193 (47.2) & $\begin{array}{c}10.0 \\
(9.6-10.5)\end{array}$ & $\begin{array}{c}21.4 \\
(20.0-22.7)\end{array}$ & $\begin{array}{c}7.1 \\
(6.8-7.5)\end{array}$ & 2450 (52.8) & $\begin{array}{c}8.9 \\
(8.5-9.2)\end{array}$ & $\begin{array}{c}17.7 \\
(16.6-18.8)\end{array}$ & $\begin{array}{c}6.7 \\
(6.3-7.0)\end{array}$ \\
\hline
\end{tabular}

readmissions and deaths could be reduced with physician follow-up, particularly when the patient is followed by the physician involved in the patient's hospital care. ${ }^{48}$ Randomized trials of targeted strategies to reduce readmission in patients discharged receiving oral anticoagulant treatment are still needed and are a high priority.

Sex differences in rates of venous thromboembolic events have been previously reported, although the reasons for higher rates in males are not entirely clear. ${ }^{49-53}$ Stroke rates in patients with atrial fibrillation may not vary by sex. ${ }^{54}$ Bleeding rates among men receiving oral anticoagulant treatment, either direct-acting or warfarin, have also been reported to be higher than those in women, ${ }^{53,55}$ but this has been refuted by other investigators, ${ }^{56}$ so sex differences in hemorrhage rates in patients receiving oral anticoagulant treatment are also unclear.

\section{Limitations}

Our study has several strengths, including a large sample, validated data sources and inclusion of virtually all people aged 66 or more in a large, diverse Canadian province. Limitations include that the results cannot be generalized to younger populations, although transition from hospital care to home is problematic for children as well as adults. ${ }^{57}$ Second, minor 


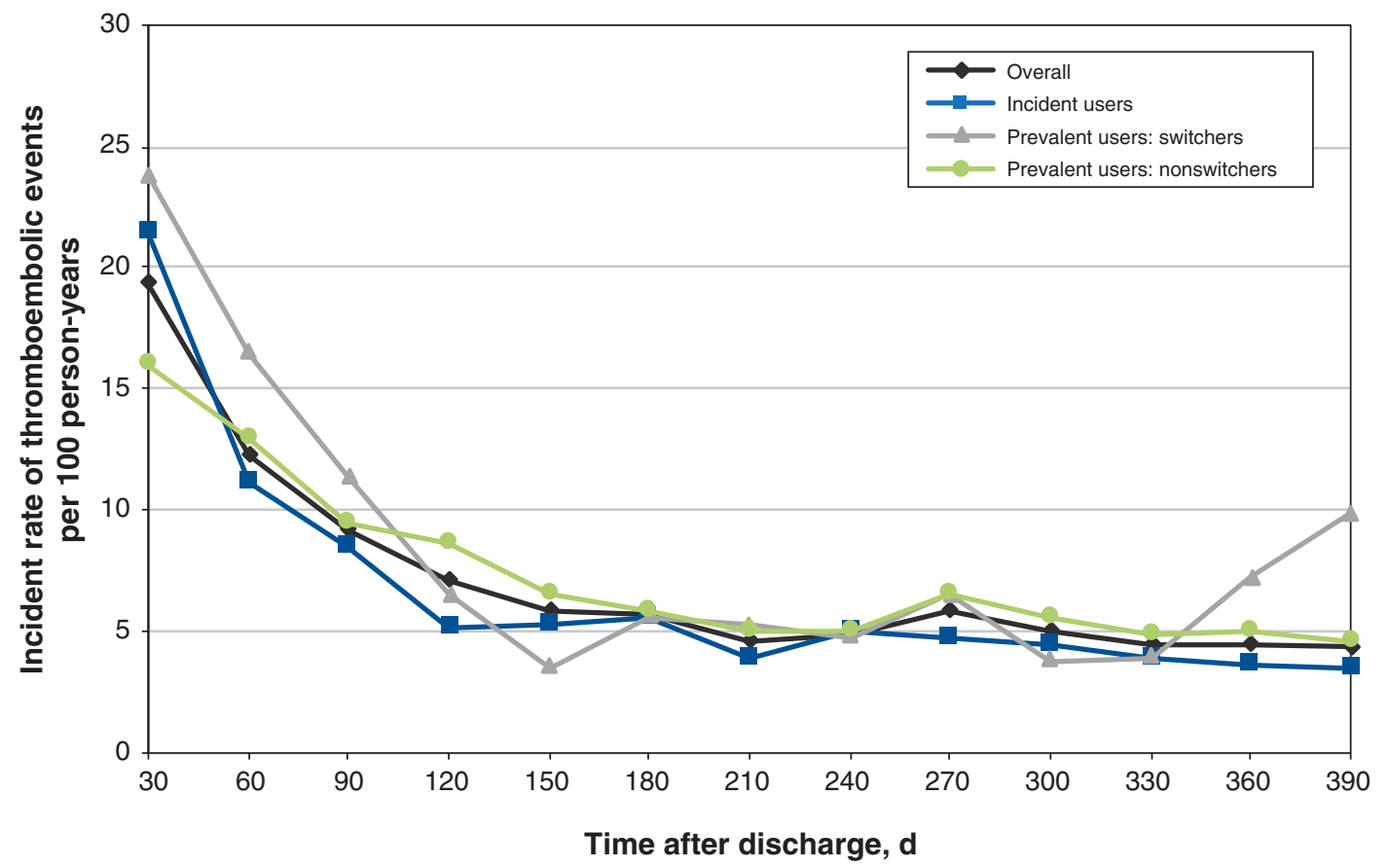

Figure 4: Postdischarge thromboembolic event rates.

events that do not lead to hospital admission or emergency department visits can still affect quality of life adversely, but are not captured in our data. Third, use of observational data collected as part of routine clinical care always entails some risk of information bias. However, missing data were rare in our study, at less than $0.07 \%$, and misclassification bias for key elements including hospital discharge, prescription dispensing, and morbid outcomes requiring hospital admission or an emergency department visit is known to be low. ${ }^{58-60} \mathrm{We}$ plan to conduct a follow-up study on predictors of our outcome events to address in more detail unmeasured confounding and death as a competing risk of outcomes. ${ }^{61}$

\section{Conclusion}

This study shows that adverse events related to oral anticoagulant treatment after hospital discharge are common among older adults in Ontario, particularly in the first 30 days after discharge. This finding supports the need for trials of organized interventions at discharge and early after discharge, as well as further analyses of predictors of adverse events related to oral anticoagulant treatment.

\section{References}

1. Cohen AT, Hamilton M, Mitchell SA, et al. Comparison of the novel oral anticoagulants apixaban, dabigatran, edoxaban, and rivaroxaban in the initial and long-term treatment and prevention of venous thromboembolism: systematic review and network meta-analysis. PLoS One 2015;10:e144856.

2. Kearon C, Akl EA, Ornelas J, et al. Antithrombotic therapy for VTE disease: CHEST guideline and expert panel report. Chest 2016;149:315-52.
3. Macle L, Cairns J, Leblanc K, et al. 2016 focused update of the Canadian Cardiovascular Society guidelines for the management of atrial fibrillation. Can 7 Cardiol 2016;32:1170-85.

4. Ruff CT, Giugliano RP, Braunwald E, et al. Comparison of the efficacy and safety of new oral anticoagulants with warfarin in patients with atrial fibrillation: a meta-analysis of randomised trials. Lancet 2014;383:955-62.

5. Wells G, Coyle D, Cameron C, et al. Safety, effectiveness, and costeffectiveness of new oral anticoagulants compared with warfarin in preventing stroke and other cardiovascular events in patients with atrial fibrillation. Ottawa: Canadian Agency for Drugs and Technologies in Health; 2012.

6. White RH. The epidemiology of venous thromboembolism. Circulation 2003; 107(Suppl 1):I4-8.

7. Bai Y, Guo SD, Deng H, et al. Effectiveness and safety of oral anticoagulants in older patients with atrial fibrillation: a systematic review and metaregression analysis. Age Ageing 2018;47:9-17.

8. Kirley K, Qato Dima M, Kornfield R, et al. National trends in oral anticoagulant use in the United States, 2007 to 2011. Circ Cardiovasc Qual Outcomes 2012; 5:615-21.

9. Weitz JI, Semchuk W, Turpie AG, et al. Trends in prescribing oral anticoagulants in Canada, 2008-2014. Clin Ther 2015;37:2506-14.e4.

10. Tsai AW, Cushman M, Rosamond WD, et al. Cardiovascular risk factors and venous thromboembolism incidence: the longitudinal investigation of thromboembolism etiology. Arch Intern Med 2002;162:1182-9.

11. Tu JV, Nardi L, Fang J, et al. National trends in rates of death and hospital admissions related to acute myocardial infarction, heart failure and stroke, 1994-2004. CMA7 2009; 180:E118-25.

12. Sargar $\mathrm{S}$. Treatment of AF in the very old patient: Do comorbidities play a role in drug choice? e-7 Cardiol Pract 2019;17:May 15.

13. Medication reconciliation in acute care: getting started kit. Version 4. Canadian Patient Safety Institute/ISMP; 2017. Available: www.ismp-canada. org/download/MedRec/MedRec-AcuteCare-GSK-EN.pdf (accessed 2017 Nov. 10).

14. Bayoumi I, Dolovich L, Hutchison B, et al. Medication-related emergency department visits and hospitalizations among older adults. Can Fam Physician 2014;60:e217-22.

15. Budnitz DS, Lovegrove MC, Shehab N, et al. Emergency hospitalizations for adverse drug events in older Americans. N Engl f Med 2011;365:2002-12.

16. Patel TK, Patel PB. Mortality among patients due to adverse drug reactions that lead to hospitalization: a meta-analysis. Eur 7 Clin Pharmacol 2018;74: 819-32. 
17. Krumholz HM. Post-hospital syndrome - an acquired, transient condition of generalized risk. N Engl F Med 2013;368:100-2

18. Hanlon JT, Pieper CF, Hajjar ER, et al. Incidence and predictors of all and preventable adverse drug reactions in frail elderly persons after hospital stay. 7 Gerontol A Biol Sci Med Sci 2006;61:511-5.

19. Parekh N, Ali K, Stevenson JM, et al. Incidence and cost of medication harm in older adults following hospital discharge: a multicentre prospective study in the UK. Br 7 Clin Pharmacol 2018;84:1789-97.

20. Gomes T, Mamdani MM, Holbrook AM, et al. Rates of hemorrhage during warfarin therapy for atrial fibrillation. CMA7 2013;185:E121-7.

21. von Elm E, Altman DG, Egger M, et al. The Strengthening the Reporting of Observational Studies in Epidemiology (STROBE) statement: guidelines for reporting observational studies. Epidemiology 2007;18:800-4.

22. Langan SM, Schmidt SA, Wing K, et al. The REporting of studies Conducted using Observational Routinely collected health Data statement for Pharmacoepidemiology (RECORD-PE). BMF 2018;363:k3532.

23. ICES data. Toronto: ICES. Available: www.ices.on.ca/Data-and-Privacy/ ICES-data (accessed 2020 Jan. 26).

24. Schultz SE, Rothwell DM, Chen Z, et al. Identifying cases of congestive heart failure from administrative data: a validation study using primary care patient records. Chronic Dis Inj Can 2013;33:160-6.

25. Lipscombe LL, Hwee J, Webster L, et al. Identifying diabetes cases from administrative data: a population-based validation study. BMC Health Serv Res 2018;18:316.

26. Quan H, Khan N, Hemmelgarn BR, et al. Validation of a case definition to define hypertension using administrative data. Hypertension 2009;54:1423-8.

27. How we collect cancer registry data. Toronto: Cancer Care Ontario. Available: www.cancercareontario.ca/en/data-research/accessing-data/technical-information/ cancer-registry-data-collection (accessed 2020 Jan. 26).

28. Fleet JL, McArthur E, Patel A, et al. Risk of rhabdomyolysis with donepezil compared with rivastigmine or galantamine: a population-based cohort study. CMA7 2019;191:E1018-24.

29. Piran S, Schulman S. Treatment of bleeding complications in patients on anticoagulant therapy. Blood 2019;133:425-35.

30. Tamariz L, Harkins T, Nair V. A systematic review of validated methods for identifying venous thromboembolism using administrative and claims data. Pharmacoepidemiol Drug Saf 2012;21(Suppl 1):154-62.

31. Sanfilippo KM, Wang TF, Gage BF, et al. Improving accuracy of International Classification of Diseases codes for venous thromboembolism in administrative data. Thromb Res 2015;135:616-20.

32. Arnason T, Wells PS, van Walraven C, et al. Accuracy of coding for possible warfarin complications in hospital discharge abstracts. Thromb Res 2006;118: 253-62.

33. White RH, Garcia M, Sadeghi B, et al. Evaluation of the predictive value of ICD-9-CM coded administrative data for venous thromboembolism in the United States. Thromb Res 2010;126:61-7.

34. Austin PC, Daly PA, Tu JV. A multicenter study of the coding accuracy of hospital discharge administrative data for patients admitted to cardiac care units in Ontario. Am Heart 7 2002;144:290-6.

35. Tu K, Mitiku T, Guo H, et al. Myocardial infarction and the validation of physician billing and hospitalization data using electronic medical records. Chronic Dis Can 2010;30:141-6.

36. Alotaibi GS, Wu C, Senthilselvan A, et al. The validity of ICD codes coupled with imaging procedure codes for identifying acute venous thromboembolism using administrative data. Vasc Med 2015;20:364-8.

37. Deyo RA, Cherkin DC, Ciol MA. Adapting a clinical comorbidity index for use with ICD-9-CM administrative databases. 7 Clin Epidemiol 1992; 45:613-9.

38. Hux JE, Ivis F, Flintoft V, et al. Diabetes in Ontario: determination of prevalence and incidence using a validated administrative data algorithm. Diabetes Care 2002;25:512-6.

39. Lip GY, Nieuwlaat R, Pisters R, et al. Refining clinical risk stratification for predicting stroke and thromboembolism in atrial fibrillation using a novel risk factor-based approach: the Euro Heart Survey on Atrial Fibrillation. Chest 2010;137:263-72

40. Tu K, Campbell NR, Chen ZL, et al. Accuracy of administrative databases in identifying patients with hypertension. Open Med 2007;1:e18-26.

41. Pisters R, Lane DA, Nieuwlaat R, et al. A novel user-friendly score (HASBLED) to assess 1-year risk of major bleeding in patients with atrial fibrillation: the Euro Heart Survey. Chest 2010;138:1093-100.

42. Klarenbach S, Lee K, Boucher M, et al. Direct oral anticoagulants for the treatment of venous thromboembolic events: economic evaluation. Ottawa: Canadian Agency for Drugs and Technologies in Health; 2016.

43. Agarwal S, Hachamovitch R, Menon V. Current trial-associated outcomes with warfarin in prevention of stroke in patients with nonvalvular atrial fibrillation: a meta-analysis. Arch Intern Med 2012;172:623-31.

44. Gallagher AM, van Staa TP, Murray-Thomas T, et al. Population-based cohort study of warfarin-treated patients with atrial fibrillation: incidence of cardiovascular and bleeding outcomes. BM7 Open 2014;4:e003839.

45. Giardina C, Cutroneo PM, Mocciaro E, et al. Adverse drug reactions in hospitalized patients: results of the FORWARD (Facilitation of Reporting in Hospital Ward) study. Front Pharmacol 2018;9:350.
46. Kanaan AO, Donovan JL, Duchin NP, et al. Adverse drug events after hospital discharge in older adults: types, severity, and involvement of Beers criteria medications. 7 Am Geriatr Soc 2013;61:1894-9.

47. Leppin AL, Gionfriddo MR, Kessler M, et al. Preventing 30-day hospital readmissions: a systematic review and meta-analysis of randomized trials. 7AMA Intern Med 2014;174:1095-107.

48. van Walraven C, Mamdani M, Fang J, et al. Continuity of care and patient outcomes after hospital discharge. 7 Gen Intern Med 2004;19:624-31.

49. Keenan CR, White RH. The effects of race/ethnicity and sex on the risk of venous thromboembolism. Curr Opin Pulm Med 2007;13:377-83.

50. McRae S, Tran H, Schulman S, et al. Effect of patient's sex on risk of recurrent venous thromboembolism: a meta-analysis. Lancet 2006;368:371-8.

51. Roach REJ, Cannegieter SC, Lijfering WM. Differential risks in men and women for first and recurrent venous thrombosis: the role of genes and environment. 7 Thromb Haemost 2014;12:1593-600.

52. Raccah BH, Perlman A, Zwas DR, et al. Gender differences in efficacy and safety of direct oral anticoagulants in atrial fibrillation: systematic review and network meta-analysis. Ann Pharmacother 2018;52:1135-42.

53. Penttilä T, Lehto M, Niiranen J, et al. Differences in the risk of stroke, bleeding events, and mortality between female and male patients with atrial fibrillation during warfarin therapy. Eur Heart 7 Cardiovasc Pharmacother 2019;5:29-36.

54. Renoux C, Coulombe J, Suissa S. Revisiting sex differences in outcomes in non-valvular atrial fibrillation: a population-based cohort study. Eur Heart $f$ 2017;38:1473-9.

55. Lapner S, Cohen N, Kearon C. Influence of sex on risk of bleeding in anticoagulated patients: a systematic review and meta-analysis. 7 Thromb Haemost 2014;12:595-605.

56. Souverein, PC, van den Ham, HA, Huerta, C, et al. Comparing risk of major bleeding between users of different oral anticoagulants in patients with nonvalvular atrial fibrillation. Br 7 Clin Pharmacol 2021;87:988-1000.

57. Auger KA, Kenyon CC, Feudtner C, et al. Pediatric hospital discharge interventions to reduce subsequent utilization: a systematic review. $7 \mathrm{Hosp} \mathrm{Med}$ 2014;9:251-60.

58. Juurlink DPC, Croxford R, Chong A, et al. Canadian Institute for Health Information Discharge Abstract Database: a validation study. Toronto: Institute for Clinical Evaluative Sciences; 2006. Available: https://www.ices. on.ca/flip-publication/canadian-institute-for-health-information-discharge/ files/assets/ basic-html/index.html\#1 (accessed 2018 Aug. 1).

59. Levy AR, O'Brien BJ, Sellors C, et al. Coding accuracy of administrative drug claims in the Ontario Drug Benefit database. Can 7 Clin Pharmacol 2003; 10:67-71.

60. Williams JI, Young W. A summary of studies on the quality of health care administrative databases in Canada. In: Goel V, Williams JI, Anderson GM, et al., editors. Patterns of health care in Ontario. The ICES practice atlas. $2 \mathrm{nd}$ ed. Ottawa: Canadian Medical Association; 1996:339-46.

61. Benipal H, Holbrook A, Paterson JM, et al. Predictors of oral anticoagulantassociated adverse events in seniors transitioning from hospital to home: a retrospective cohort study protocol. BM7 Open 2020;10:e036537.

Affiliations: Division of Clinical Pharmacology \& Toxicology (Holbrook), Department of Medicine and Department of Health Research Methods, Evidence, and Impact (Holbrook, Benipal, Greaves), McMaster University, Hamilton, Ont.; ICES (Paterson, Gomes); Institute of Health Policy, Management and Evaluation (Paterson), University of Toronto, Toronto, Ont.; Schulich School of Medicine and Dentistry (Lee), Western University, London, Ont.; Li Ka Shing Knowledge Institute (Martins, Gomes), St. Michael's Hospital, Toronto, Ont.

Contributors: Anne Holbrook conceived the study. Anne Holbrook, Michael Paterson, Diana Martins, Simon Greaves and Tara Gomes designed the study. Anne Holbrook and Harsukh Benipal drafted the manuscript. All of the authors contributed to data acquisition, analysis and interpretation, revised the manuscript for important intellectual content, approved the final version to be published and agreed to be accountable for all aspects of the work.

Funding: This study was funded by grants to Anne Holbrook from the Hamilton Academic Health Science Centre Alternative Funding Plan Innovation Fund (HAH-16-06) and from the Canadian Institutes for Health Research (365834). It was also supported by the Ontario Drug Policy Research Network, which is funded by grants from the Ontario Ministry of Health $(\mathrm{MOH})$, and by ICES, which is funded by an annual grant from the Ontario $\mathrm{MOH}$.

Content licence: This is an Open Access article distributed in accordance with the terms of the Creative Commons Attribution (CC BYNC-ND 4.0) licence, which permits use, distribution and reproduction in any medium, provided that the original publication is properly cited, the use is noncommercial (i.e., research or educational use), and no 
modifications or adaptations are made. See: https://creativecommons. org/licenses/by-nc-nd/4.0/.

Data sharing: The data set from this study is held securely in coded form at ICES. Although data-sharing agreements prohibit ICES from making the data set publicly available, access may be granted to those who meet prespecified criteria for confidential access, available at https://www.ices. on.ca/DAS. The underlying analytic code is available from the authors on request, with the understanding that the computer programs may rely on coding templates or macros that are unique to ICES and are therefore inaccessible or may require modification.
Disclaimer: Parts of this material are based on data and information compiled and provided by the Ontario Ministry of Health, the Canadian Institute for Health Information, Cancer Care Ontario and IQVIA Canada. The analyses, conclusions, opinions and statements expressed herein are those of the authors and do not reflect those of the funding or data sources; no endorsement is intended or should be inferred.

Supplemental information: For reviewer comments and the original submission of this manuscript, please see www.cmajopen.ca/content/9/2/ E364/suppl/DC1. 